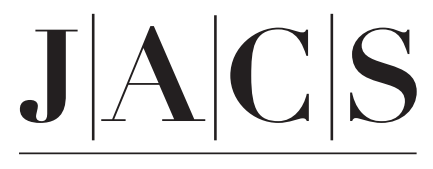

A R T I C L E S

Published on Web 08/11/2005

\title{
Increase of the Photoluminescence Intensity of InP Nanowires by Photoassisted Surface Passivation
}

\author{
Lambert K. van Vugt, ${ }^{* \dagger}$ Sandra J. Veen, ${ }^{\dagger}$ Erik P. A. M. Bakkers, ${ }^{\ddagger}$ \\ Aarnoud L. Roest, $\S^{\S}$ and Daniël Vanmaekelbergh ${ }^{\dagger}$ \\ Contribution from the Debye Institute, Condensed Matter and Interfaces, Utrecht University, \\ Post Office Box 80 000, 3508 TA Utrecht, The Netherlands, Philips Research Laboratories, \\ Professor Holstlaan 4, 5656 AA Eindhoven, The Netherlands, and Kavli Institute of \\ Nanoscience, Delft University of Technology, Lorentzweg 12678 JK Delft, The Netherlands
}

Received March 23, 2005; E-mail: L.K.vanVugt@Phys.uu.nl

\begin{abstract}
As-grown single-crystal InP nanowires, covered with a surface oxide, show a photoluminescence efficiency that strongly varies from wire to wire. We show that the luminescence efficiency of single-crystal InP nanowires can be improved by photoassisted wet chemical etching in a butanol solution containing HF and the indium-coordinating ligand trioctylphosphine oxide. Electron-hole photogeneration, electron scavenging, and oxidative dissolution combined with surface passivation by the indium-coordinating ligand are essential elements to improve the luminescence efficiency. Time traces of the luminescence of surfacepassivated wires show strong oscillations resembling the on-off blinking observed with single quantum dots. These results reflect the strong influence of a single or a few nonradiative recombination center(s) on the luminescence properties of an entire wire.
\end{abstract}

\section{Introduction}

Semiconductor nanowires form, perhaps, the most promising class of nanocrystalline materials for future application in miniaturized optoelectronic devices and photonic circuits. ${ }^{1-3}$ Single-crystal nanorods and wires of many group IV, III-V, and II-VI compound semiconductors ${ }^{4}$ can be grown by the vapor-liquid-solid (VLS) ${ }^{5}$ method, using a metal droplet as catalyst. The large length-to-diameter aspect ratio enables the wires to be contacted by standard lithographic procedures, ${ }^{6}$ incorporating them into an electronic or optoelectronic device. For instance, an electroluminescent device based on a $\mathrm{p}-\mathrm{n}$ junction formed by two crossed InP nanowires has been reported. ${ }^{2}$ Furthermore, nanowires with a built-in $\mathrm{p}-\mathrm{n}$ junction ${ }^{3}$ or a zero-dimensional quantum $\operatorname{dot}^{7,8}$ have been synthesized. Such systems are very promising for nanoscale optoelectronic devices with novel functions, such as the emission of polarized photons and single-photon pulses. For such applications it is required that the semiconductor nanowires have a reasonable

\footnotetext{
$\dagger$ Utrecht University.

$\doteqdot$ Philips Research Laboratories.

$\S$ Delft University of Technology.

(1) Hiruma, K.; Yazawa, M.; Katsuyama, T.; Ogawa, K.; Haraguchi, K.; Koguchi, M.; Kakibayashi, H. J. Appl. Phys. 1995, 77, 447-462.

(2) Duan, X.; Huang, Y.; Cui, Y.; Wang, J.; Lieber, C. M. Nature 2001, 409 $66-69$.

(3) Gudiksen, M. S.; Lauhon, L. J.; Wang, J.; Smith, D. C.; Lieber, C. M. Nature 2002, 415, 617-620.

(4) Duan, X.; Lieber, C. M. Adv. Mater. 2000, 12, 298-302.

(5) Wagner, R. S.; Ellis, W. C. Appl. Phys. Lett. 1964, 4, 89-90.

(6) Tans, S. J.; Verschueren, A. R. M.; Dekker, C. Nature 1998, 393, 49-52.

(7) Björk, M. T.; Ohlsson, B. J.; Sass, T.; Persson, A. I.; Thelander, C. Magnussson, M. H.; Deppert, K.; Wallenberg, L. R.; Samuelson, L. Appl. Phys. Lett. 2002, 80, 1058-1060.

(8) Wu, Y.; Fan, R.; Yang, P. Nano Lett. 2002, 2, 83-86.
}

10.1021/ja051860o CCC: $\$ 30.25$ @ 2005 American Chemical Society electron-to-photon or photon-to-photon luminescence quantum yield. Semiconductor nanowires can be grown as (nearly) defectfree single crystals. ${ }^{9}$ They, however, possess a relative large surface-to-volume ratio. As a consequence, excitons can decay nonradiatively via electronic surface states, leading to a poor photoluminescence quantum yield.

InP, the semiconductor of interest in the present study, has a direct band gap of $1.38 \mathrm{eV}$ at room temperature. Light-emitting diodes based on macroscopic $\mathrm{p}-\mathrm{n}$ junctions of InP (as base material) show a charge carrier-to-photon conversion efficiency of nearly $100 \% .{ }^{10}$ In strong contrast, an InP nanowire with a $\mathrm{p}-\mathrm{n}$ junction shows an efficiency of $\sim 0.1 \% .^{3}$ In principle, nonradiative recombination can occur at the surface or in the bulk. For instance, Au atoms can be incorporated into the lattice during growth of the wire, leading to a quenching state in the band gap. The solubility of $\mathrm{Au}$ atoms in InP is, however, very low $\left(6 \times 10^{14} / \mathrm{cm}^{3}\right),{ }^{11}$ which indicates that in the case of wires it cannot be the main origin of nonradiative recombination. The disappointingly low quantum yield is in line with the results obtained with suspensions of spherical InP nanocrystals (socalled quantum dots), where the photoluminescence efficiency of the as-prepared colloidal quantum dots is below $1 \%$. However, Mićić et al. reported an effective surface passivation procedure based on the use of a solution of butanol/HF/

(9) Krishnamachari, U.; Borgstrom, M.; Ohlsson, B. J.; Panev, N.; Samuelson, L.; Seifert, W.; Larsson, M. W.; Wallenberg, L. R. Appl. Phys. Lett. 2004, 85, 2077-2079.

(10) Steigerwald, D. A.; Bhat, J. C.; Collins, D.; Fletcher, R. M.; Holcomb, M O.; Ludowise, M. J.; Martin, P. S.; Rudaz, S. L. IEEE J. Sel. Top. Quantum Electron. 2002, 8, 310-320.

(11) Parguel, V.; Favennec, P. N.; Gauneau, M.; Rihet, Y.; Chaplain, R.; L'Haridon, H.; Vaudry, C. J. Appl. Phys. 1987, 62, 824-827.

J. AM. CHEM. SOC. 2005, 127, 12357-12362 - 12357 
trioctylphosphine oxide (TOPO) which increased the photoluminescence quantum yield to $40 \% .^{12}$ Recently, Talapin et al. showed convincingly that the surface passivation of InP nanocrystals is photochemical in nature. ${ }^{13}$ The surface passivation requires oxidative dissolution of InP surface molecules, induced by photogenerated valence holes, together with the action of HF and the TOPO capping molecules. Although the molecular mechanism of the surface passivation is not understood in detail, it was reported that the increased photoluminescence is directly related to a decrease of unpassivated $\mathrm{P}$ sites and an increase of In-TOPO sites at the nanocrystal surface. ${ }^{14}$

It is obvious that the photoluminescence and electroluminescence quantum yield of InP nanowires must be considerably increased before study of their fundamental photophysics and their application in optoelectronic devices becomes feasible. In the present study, we have investigated whether the lightstimulated surface passivation procedure, proven to increase efficiently the photoluminescence quantum yield of InP quantum dot suspensions, can also be used for InP nanowires. There are some differences between wires and colloidal nanocrystals which must be considered here. First, the length of the InP nanowires that we have studied exceeds the exciton Bohr radius in InP $(\sim 20 \mathrm{~nm})$ by at least 1 order of magnitude, while the diameter of the wires is between 30 and $60 \mathrm{~nm}$. Since quantumconfinement effects could be expected in these wires only for diameters below $15 \mathrm{~nm},{ }^{15}$ such effects can be neglected here. But importantly, highly polarized photoluminescence has been observed from these one-dimensional structures. ${ }^{16}$ In addition, several independent excitonic states can coexist in a single wire, unlike the case of an InP quantum dot. This is important for the photophysics and photochemical surface passivation of the InP nanowires under study. Second, the absolute number of surface atoms of a single-crystal InP wire is much larger than that of an InP quantum dot. Thus, the probability that a surface defect, inducing nonradiative recombination, is present on a wire is considerably larger than that on a quantum dot. All excitons located within a distance shorter than the diffusion length (which can be $100 \mathrm{~nm}$ or more) from a surface defect will be annihilated nonradiatively. In other words, a single defect site can strongly affect the luminescence properties of a single wire. It is thus an intriguing question as to whether single nanowires can be efficiently passivated against nonradiative recombination. In this respect, the case of a nanowire is similar to that of a quantumdot solid, where due to efficient energy transfer, a single nonradiative center can quench the luminescence. ${ }^{17}$

Here, we present a study of the light-stimulated surface passivation and photoetching of InP nanowires. We have studied the photoluminescence spectra of individual $\mathrm{InP}$ nanowires deposited on $\mathrm{Si} / \mathrm{SiO}_{2}$ substrates and (3-aminopropyl)triethoxysilane (APTES)-covered substrates before and after photoetching in HF/TOPO solutions in butanol. In addition, we have imaged individual InP nanowires and recorded the evolution

(12) Mićić, O. I.; Sprague, J.; Lu, Z.; Nozik, A. J. Appl. Phys. Lett. 1996, 68, 3150-3152.

(13) Talapin, D. V.; Gaponik, N.; Borchert, H.; Rogach, A. L.; Haase, M.; Weller, H. J. Phys. Chem. B 2002, 106, 12659-12663.

(14) Adam, S.; McGinley, C.; Möller, T.; Talapin, D. V.; Borchert, H.; Haase, M.; Weller, H. Eur. Phys. J. D 2003, 24, 373-376.

(15) Gudiksen, M. S.; Wang, J.; Lieber, C. M. J. Phys. Chem. B 2002, 106 4036-4039.

(16) Wang, J.; Gudiksen, M. S.; Duan, X.; Cui, Y.; Lieber, C. M. Science 2001 $293,1455-1457$.

(17) Kagan, C. R.; Murray, C. B.; Nirmal, M.; Bawendi, M. G. Phys. Rev. Lett. 1996, 76, 1517-1520 of the photoluminescence during the process of photoetching (at high HF concentration) or photostimulated surface passivation (at low HF concentration) using nonpolarized and linearly polarized excitation light.

\section{Experimental Section}

Materials. Chlorobenzene (99.9\%; HPLC grade), trioctylphosphine oxide (TOPO; 99\%), butanol (99.8\%; HPLC grade), and (3-aminopropyl)triethoxysilane (APTES; 99\%) were purchased from Aldrich. Hydrofluoric acid (40\% in water) was purchased from Riedel-Dehaen. Hydrogen peroxide (30\% in water) was purchased from Merck.

Nanowire Synthesis and Substrate Preparation. The n-type InP nanowires ${ }^{18}$ were synthesized by a laser ablation method. An InP:Se target was placed upstream of an 2 - $\AA$ gold-covered $\mathrm{Si} / \mathrm{SiO}_{2}$ substrate inside a tube oven. Ablation for $30 \mathrm{~min}$ in a flow of argon resulted in $\sim 10-\mu \mathrm{m}$-long and, on average, 50 -nm-thick nanowires. ${ }^{19,20}$ The asgrown nanowires were dispersed in chlorobenzene by ultrasonification and deposited onto bare or APTES-functionalized $\mathrm{Si} / \mathrm{SiO}_{2}$ substrates. Markers on the substrates, defined by e-beam lithography, allowed repeated identification of individual nanowires.

For in situ microluminescence measurements, a drop of the etching solution (5 g/L TOPO, $0.01-20 \%$ HF in butanol) ${ }^{21}$ was placed on top of the nanowire-containing substrate, which was located inside a Teflon holder fitted with a sapphire window.

Microluminescence Setup. Single-wire photoluminescence measurements were performed using a linearly polarized 200-mW, 457nm DPSS laser (Melles Griot) directly coupled into a Leica DM/LM upright microscope equipped with $5-100 \times$ dry $\mathrm{BF} / \mathrm{DF}$ objectives to have a resulting magnification of $50-1000 \times$. A $\lambda / 2$ wave retardation plate was placed directly after the laser to enable rotation of the light polarization in the specimen plane of the microscope. The photoluminescence signal of the nanowires was split off using a long-pass dichroic mirror and blocking filter. This signal was then coupled into an optical fiber, dispersed by a grating, and recorded by a CCD array spectrometer. Images were acquired by placing a CCD camera (Nikon DM12) directly on top of the microscope.

Ex Situ Photoetch Setup. White light from a 300-W $\mathrm{Hg} / \mathrm{Xe}$ lamp (Oriel) was focused by a lens and deflected downward by a mirror into an open $50-\mathrm{mL}$ polypropylene vial which was placed on top of a magnetic stirrer.

TEM. Nanowires were deposited on a $10-\mathrm{nm}$-thick $\mathrm{Si}_{3} \mathrm{~N}_{4}$ membrane, such that specific wires could be studied before and after the etching experiment. TEM images were obtained with a Philips Tecnai 12 instrument operating at $120 \mathrm{kV}$.

\section{Results and Discussion}

3.1. Photoluminescence Spectra of As-Grown and SurfacePassivated InP Nanowires. Figure 1A shows typical PL spectra of an as-grown InP wire and of the same wire after $25 \mathrm{~min}$ of etching in a $0.1 \% \mathrm{HF} / 5 \mathrm{~g} / \mathrm{L}$ TOPO/butanol solution using defocused laser light $\left(442 \mathrm{~W} / \mathrm{cm}^{2}\right)$. Photostimulated passivation of this wire resulted in a 10-fold increase of the PL intensity (see below). Figure 1B,C shows the dark-field optical image of the wire and the resulting PL image after photoetching. This shows that the photoluminescence intensity is uniform along the length of the wire. The maximum of the photoemission of the as-grown wire is noticeably blue-shifted $(59 \mathrm{meV})$ with

(18) De Franceschi, S.; van Dam, J. A.; Bakkers, E. P. A. M.; Feiner, L. F.; Gurevich, L.; Kouwenhoven, L. P. Appl. Phys. Lett. 2003, 83, 344-347. (19) Bakkers, E. P. A. M.; Verheijen, M. A. J. Am. Chem. Soc. 2003, 125, $3440-3441$

(20) Bakkers, E. P. A. M.; van Dam, J. A.; De Franceschi, S.; Kouwenhoven, L. P.; Kaiser, M.; Verheijen, M.; Wondergem, H.; van der Sluis, P. Nat. Mater. 2004, 3, 769-773.

(21) We advise those working with HF solutions to familiarize themselves with its inherent hazards. 


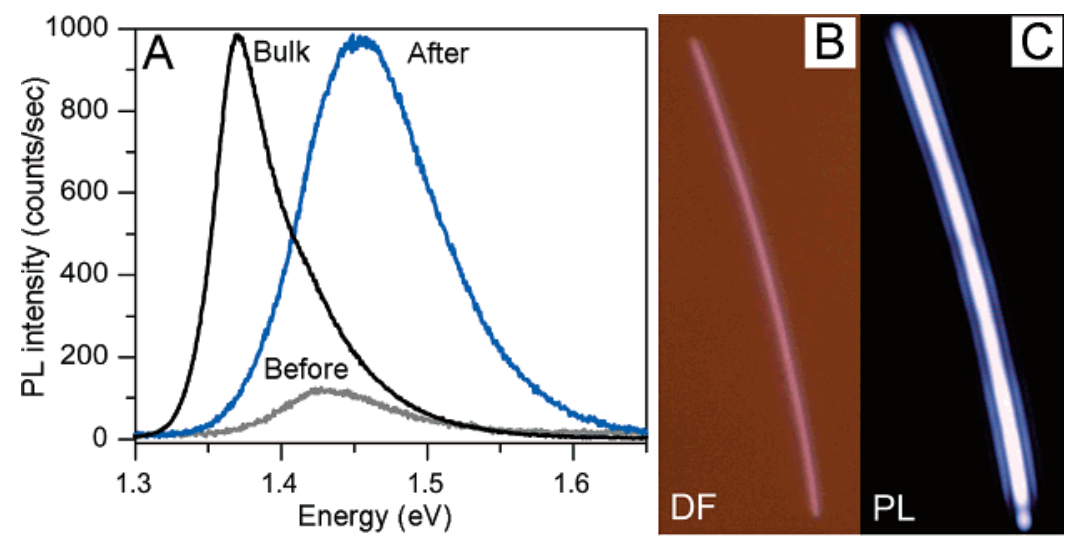

Figure 1. Photoluminescence spectra of a single nanowire before (gray curve) and after (blue curve) photoetching with defocused laserlight $\left(442 \mathrm{~W} / \mathrm{cm}^{2}\right.$ ) for $25 \mathrm{~min}$ in a $0.1 \% \mathrm{HF} / 5 \mathrm{~g} / \mathrm{L} \mathrm{TOPO/butanol} \mathrm{solution.} \mathrm{For} \mathrm{comparison,} \mathrm{the} \mathrm{normalized} \mathrm{PL} \mathrm{spectrum} \mathrm{of} \mathrm{a} \mathrm{macroscopic} \mathrm{InP:Se} \mathrm{crystal} \mathrm{is} \mathrm{plotted} \mathrm{(black}$ curve). Panels B and C show the dark-field optical and photoluminescence images of this wire, respectively.
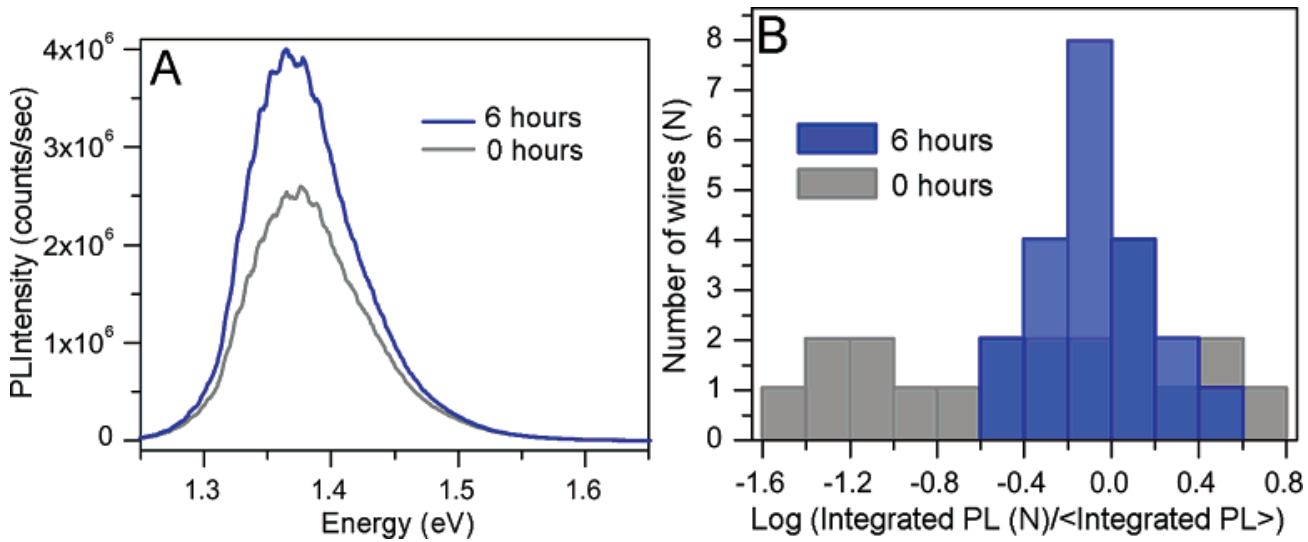

Figure 2. (A) Averaged photoluminescence intensity of an ensemble of individually measured nanowires before (gray line) and after (blue line) $6 \mathrm{~h}$ of photoetching in a $0.01 \% \mathrm{HF} / 50 \mathrm{~g} / \mathrm{L} \mathrm{TOPO} / \mathrm{butanol}$ solution under illumination of a $300-\mathrm{W}$ mercury/xenon lamp. Twenty-five wires were measured. The two highest and lowest intensities were disregarded. (B) Histogram of the luminescence intensity of individual wires: on the $x$-axis, the logarithm of the integrated luminescence intensity normalized to the ensemble average is used.

respect to that of a bulk InP:Se crystal recorded with the same apparatus. After photoetching, the maximum is further blueshifted by $40 \mathrm{meV}$. We should note here that the diameter of the wire was about $50 \mathrm{~nm}$, excluding quantum confinement as the origin of the blue-shifts. Another striking feature is that the emission spectra of the wire are considerably broader (fwhm $=90 \mathrm{meV}$ ) than those of a macroscopic InP crystal (fwhm $=$ $57 \mathrm{meV}$ ), where broadening is caused by electron-phonon interactions and shallow defects. Spectra measured on at least 50 other wires, with diameters over $40 \mathrm{~nm}$, also showed the blue-shift and the broadening of the photoemission.

We attribute the broadening and the blue-shift of the PL spectra of the nanowires to Coulomb interactions of the exciton electron and hole with (fractional) charges or dipoles distributed on the surface of the nanowire. This conclusion is based on theoretical work by Franceschetti and Zunger, who showed that the sum of the Coulomb interactions between a given spectator charge and the electron and hole of the exciton is nonzero. ${ }^{22}$ As a result, a blue- or red-shift of the exciton energy can occur depending on the effective masses of the electron and hole. In the case of InP dots, the hole is more localized than the electron; the blue-shift of the spectra is explained by the presence of an effective positive charge density on the surface of the InP nanowires. The broadening of the spectra of the wires with

(22) Franceschetti, A.; Zunger, A. Phys. Rev. B 2000, 62, R16287-R16290. respect to those of macroscopic single crystals is then explained by (temporal or stationary) spatial inhomogeneities in the effective charge density on the surface of the wire. Excitons at different positions in the wire experience a variable Coulomb potential, leading to a variation in the energy of the emitted photons. We remark that broadening of the spectra of CdSe nanorods and concomitant red-shifts have been reported recently, and have been explained in a similar way. ${ }^{23}$

3.2. Photoassisted Surface Passivation of InP Nanowires in Butanol Solutions of HF/TOPO. We used a procedure for light-stimulated surface passivation of InP nanowires which is very similar to that used by Talapin et al. ${ }^{13}$ for the photoetching and passivation of colloidal InP quantum dots (see Figure 2). We recorded the PL spectra of 25 individual nanowires before any (chemical) treatment. The wires were dispersed on a $\mathrm{Si}$ / $\mathrm{SiO}_{2}$ substrate. The as-grown wires were covered with a thin native oxide layer which forms due to contact with air. Another part of the as-grown nanowires was transferred from the growth substrate into a transparent vial containing a $0.1 \% \mathrm{HF} / \mathrm{butanol}$ solution. After $20 \mathrm{~min}$, this solution was diluted with butanol, and TOPO was added to give a final concentration of $0.01 \%$ $\mathrm{HF}$ and $50 \mathrm{~g} / \mathrm{L}$ TOPO. This procedure ensured that the surface oxide was chemically etched away before the photoetching

(23) Müller, J.; Lupton, J. M.; Rogach, A. L.; Feldmann, J.; Talapin, D. V.; Weller, H. J. Phys. Rev. Lett. 2004, 93, 167402-1-167402-4. 


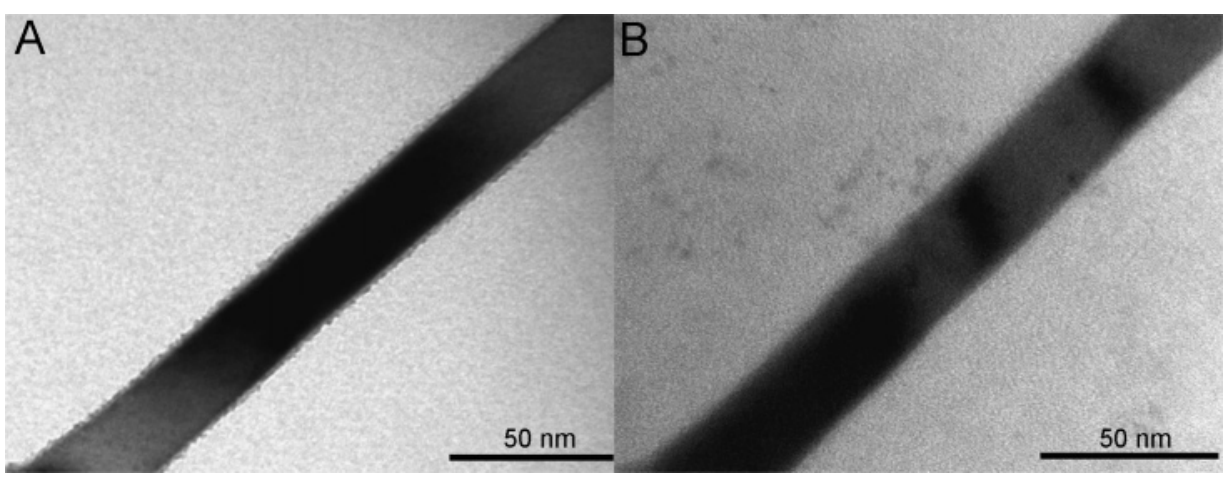

Figure 3. TEM images of a nanowire (A) before $(27.8 \pm 0.9 \mathrm{~nm}$ diameter) and (B) after $(27.1 \pm 0.2 \mathrm{~nm}$ diameter) $6 \mathrm{~h}$ of illumination with a 300 -W mercury/xenon lamp in a $0.01 \% \mathrm{HF} / 50 \mathrm{~g} / \mathrm{L}$ TOPO/butanol solution. Scale bars are $50 \mathrm{~nm}$.

began. The solution was stirred and illuminated by a $300-\mathrm{W}$ mercury/xenon lamp in ambient conditions for $6 \mathrm{~h}$. The solution was then dropcast onto a $\mathrm{Si} / \mathrm{SiO}_{2}$ substrate, washed with methanol, and dried. PL spectra of 25 randomly chosen nanowires were recorded under precisely the same conditions of focused laser light intensity as for the as-grown wires. The shape of the emission spectra of individual InP wires is very similar for all wires (see section 3.1). However, the absolute photoluminescence intensity varies strongly from wire to wire (see Figure 2B). This is very typical, both for the as-grown and for the photoetched wires, although the dispersion in luminescence intensity is smaller for the etched wires. We have estimated the PL quantum yield (QY) of the single untreated wires, by comparison of the emission intensity with that of an InP single crystal. We found that the PL QY of the single wires varied between $0.01 \%$ and $1 \%$ typically when the QY of the InP single crystal was taken as $100 \%$. To check whether photoetching has led to an improved photoluminescence, we took the average (after removal of the two highest and lowest PL intensities) of the photoluminescence spectra of 25 nanowires before and after photoetching (see Figure 2). We etched several labeled InP nanowires ranging in diameter from 26 to $50 \mathrm{~nm}$ with the same setup for $6 \mathrm{~h}$ using the same etching solutions and found no appreciable reduction of the diameter of the wire from TEM analysis (see Figure 3). This is in line with the results of Talapin et al. with InP quantum dots, which show that the removal of semiconductor material in this solution is very slow.

On average, there is an increase in the luminescence intensity (under the same excitation intensity) by a factor of 1.5 after 6 $\mathrm{h}$ of photoetching. We repeated this experiment with a series of wires from another growth run and found a similar increase in the luminescence intensity: the intensity increased by a factor of 3, averaged over 25 nanowires. Thus, prolonged photoetching with very low HF concentrations leads to a moderate increase in the averaged photoluminescence efficiency without the noticeable removal of the semiconductor material.

\subsection{Photoselectivity of Etching and Surface Passivation.}

We have further checked the nature of the processes (photochemical or chemical?) that occur when an etching solution is applied to InP wires, by comparing wires under focused laser light with wires kept in the etching solution in the dark. We also manipulate light absorption by changing the relative orientation of the E-field with respect to the wire's long axis. The as-grown nanowires are covered with an $\operatorname{In}_{2} \mathrm{O}_{3}$ and/or a mixed In/P oxide that has to be removed before InP is etched and TOPO chemisorption can take place. Oxide removal occurs

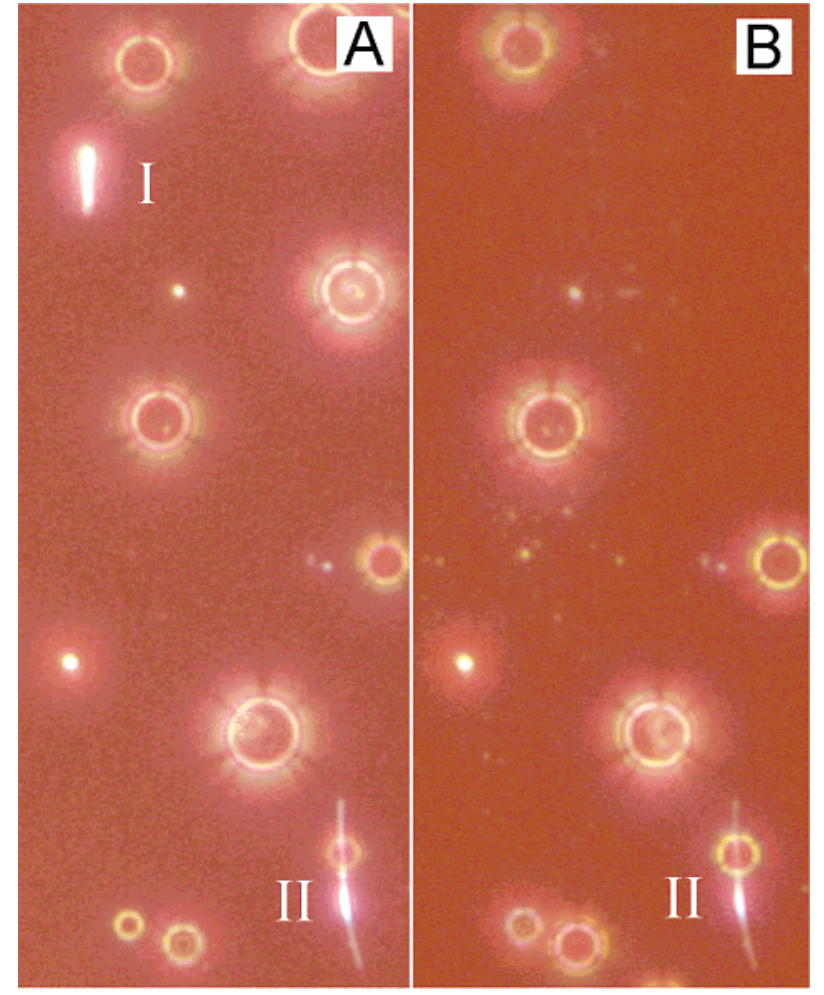

Figure 4. Optical dark-field image of two nanowires on an APTES-covered $\mathrm{SiO}_{2}$ substrate immersed in a $20 \% \mathrm{HF} /$ butanol solution. (A) After illuminating the upper wire with a focused laser beam $\left(15.9 \mathrm{~kW} / \mathrm{cm}^{2}\right)$ for $3 \mathrm{~min}$. (B) The bright circles in the pictures are caused by gas bubbles in the solution.

by chemical HF etching in the dark. To be able to see changes within a reasonable measurement time, we used focused laser light and increased the HF concentration of the etching solution to between 0.1 and $20 \% \mathrm{HF}$ in TOPO/butanol.

Figure 4A shows a low-magnification dark-field optical image of two InP nanowires in $20 \% \mathrm{HF} /$ butanol solution on an APTEScovered $\mathrm{SiO}_{2}$ substrate. Only wire I was exposed to the focused laser spot $\left(15.9 \mathrm{~kW} / \mathrm{cm}^{2}\right)$. After $3 \mathrm{~min}$ of illumination, wire I was etched away completely, while wire II remained unchanged in the dark. This clearly demonstrates that, within the time frame of the experiment, InP wires do not etch in $\mathrm{HF} /$ butanol solutions in the dark, but that photogenerated holes are required to dissolve the wire. This agrees with the observation reported by Talapin et al., that the etching of colloidal InP quantum dots in a HF/TOPO/butanol solution is photochemical. Thus, photogenerated conduction electrons are scavenged by oxygen present in the solution under ambient conditions, while the holes 

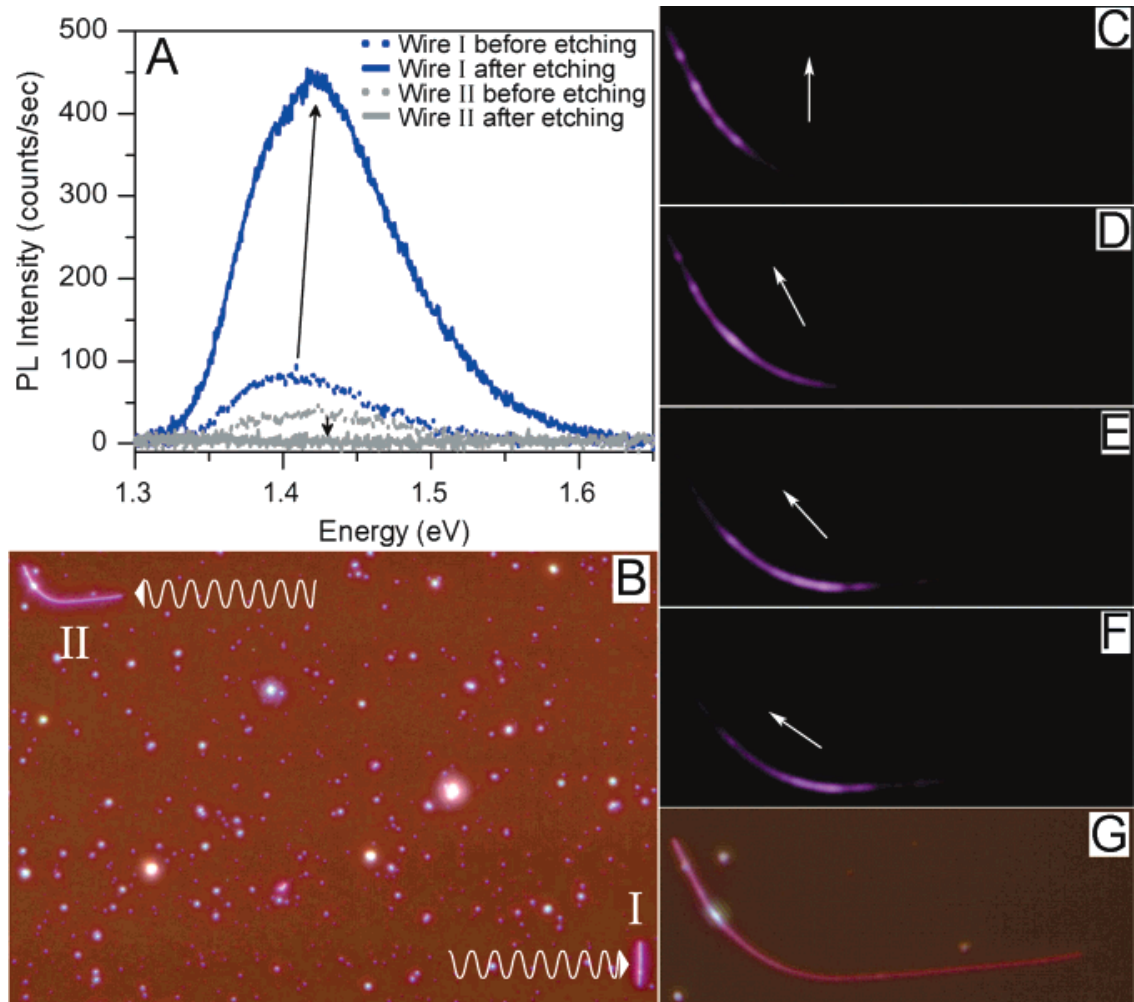

Figure 5. (A) Photoluminescence spectra of wires I and II before (dotted curves) and after (solid curves) photoetching by a defocused laser spot (442 $\mathrm{W} / \mathrm{cm}^{2}$ ) with the electric field vector parallel to wire I. The spectra were acquired with excitation polarization parallel to each wire axis. (B) Dark-field optical image of the two perpendicularly oriented nanowires. (C-F) Photoluminescence images of wire II after photoetching, showing only light emission from the bent part of the wire which could absorb the polarized light during photoetching (compare with the dark-field image $\mathrm{G}$ ). For images $\mathrm{C}-\mathrm{F}$, the excitation polarization is as indicated by the arrows. Excitation with horizontal polarization resulted in no detectable light emission.

are consumed in oxidative dissolution of InP:

$$
\begin{gathered}
6 h v \rightarrow 6 \mathrm{~h}^{+}+6 \mathrm{e}^{-} \\
\mathrm{InP}_{\text {surf }}+6 \mathrm{~h}^{+} \rightarrow \operatorname{In}(\mathrm{III})_{\mathrm{sol}}+\mathrm{P}(\mathrm{III})_{\mathrm{sol}} \\
3 /_{2} \mathrm{O}_{2}+6 \mathrm{H}^{+}+6 \mathrm{e}^{-} \rightarrow 3 \mathrm{H}_{2} \mathrm{O}
\end{gathered}
$$

Photoetch experiments have shown that the intensity of the photoemission of the InP wire did not increase in oxygen-free etch solutions; instead, the emission intensity decreased, probably due to the chemical dissolution of the surface oxide layer. The photoetching was enhanced by adding $\mathrm{H}_{2} \mathrm{O}_{2}$ as an electron scavenger to the solution. This shows that photogenerated conduction electrons must be scavenged fast enough to prevent electron-hole recombination. Furthermore, illumination of an individual nanowire in a butanol solution without $\mathrm{HF}$ or without TOPO had no effect or a negative effect on the photoluminescence intensity, showing that $\mathrm{HF}$ and TOPO are essential ingredients for photoassisted surface passivation.

We observed that when focused laser light is used, the etch rate of the wires depends on the HF concentration present in the solution. At a concentration of $20 \%$, dissolution of the entire wire with a focused laser beam is fast.

It has been shown that absorption and emission of light by InP nanowires is strongly anisotropic; ${ }^{15}$ photons polarized with their electric field vector parallel to the long axis of the wire are preferentially absorbed or emitted. This must be important in the photoetching process. In Figure 5A, two wires are shown: wire I is oriented nearly perpendicular to wire II, which has a bent part. Before the photoetching was started, luminescence spectra of both wires were taken with the electric-field vector of the incident laser light parallel to the principal axis of the wire. The photoetching was carried out in a $0.1 \% \mathrm{HF} / 5 \mathrm{~g} / \mathrm{L}$ TOPO/butanol solution with a defocused laser spot $\left(442 \mathrm{~W} / \mathrm{cm}^{2}\right)$ that illuminated both wires. During photoetching, the electric field vector was parallel to wire I. After 25 min of illumination, the sample was rinsed with butanol and dried. The spectra of the individual nanowires were taken, again with the light polarized parallel to the wire's long axis (Figure 5B). The photoluminescence intensity of wire $\mathrm{I}$ is increased by a factor of 5 due to photoetching. In contrast, the photoluminescence of wire II, which was illuminated with light perpendicular to the wire axis, has not improved. Only the bent part of the wire, which could absorb light during the photoetching, luminesces (Figure $5 \mathrm{C}-\mathrm{F}$ ). The degradation of the photoluminescence is probably due to chemical removal of the surface oxide which passivates the surface to some extent. Hence, the anisotropic light absorption enables the selective etching and passivation of a wire by a proper choice of the polarization of the incident light.

3.4. Time Evolution of the Photoluminescence of Individual Wires during Photoetching. In an attempt to get more insight into the mechanism of photoassisted surface passivation and etching and the observed variation in photoluminescence properties, we followed the photoetching of individual InP nanowires in situ. To enable direct PL measurement and imaging of the same wire before, during, and after photoetching, we deposited the as-grown nanowires on an APTES-covered $\mathrm{SiO}_{2}$ substrate. This protected the $\mathrm{SiO}_{2}$ substrate from HF attack and 

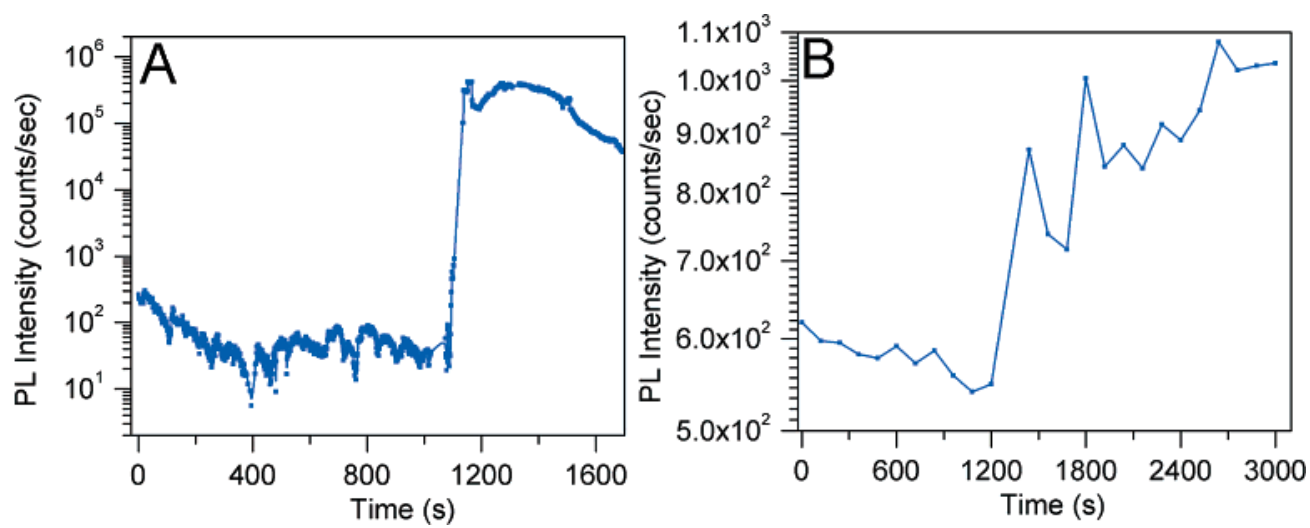

Figure 6. Time evolution of the photoluminescence from a single nanowire (A) during photoetching with a focused laser spot $\left(15.9 \mathrm{~kW} / \mathrm{cm}^{2}\right)$ in a $2.5 \%$ $\mathrm{HF} / 0.3 \mathrm{~g} / \mathrm{L}$ TOPO/butanol solution and (B) during photoetching with a focused laser spot $\left(795 \mathrm{~W} / \mathrm{cm}^{2}\right)$ in a $0.01 \% \mathrm{HF} / 0.01 \% \mathrm{H} \mathrm{O}_{2} / 6.6 \mathrm{~g} / \mathrm{L} \mathrm{TOPO} / \mathrm{butanol}^{2}$ solution.

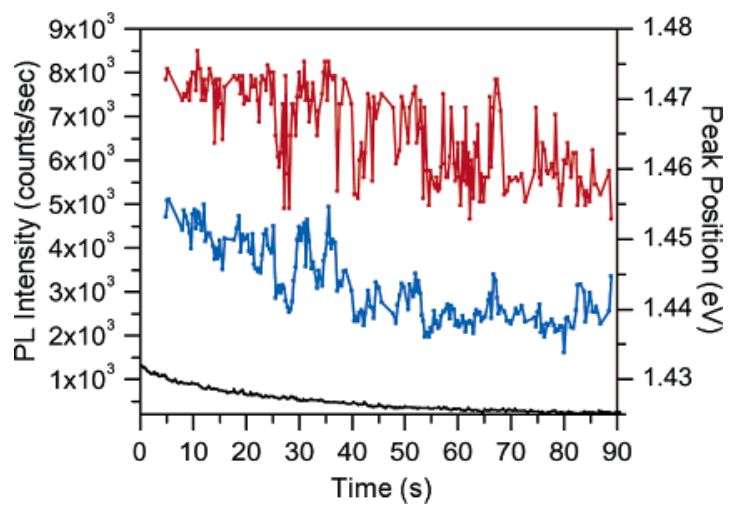

Figure 7. Fluctuations of the PL intensity (blue curve) and the peak position (red curve) of a photoetched nanowire measured under excitation with focused laser light in ambient conditions. For comparison, the PL intensity evolution of an unetched wire is also plotted (black curve).

prevented the wires from drifting. Figure 6 shows the time traces of the PL intensities of two nanowires being photoetched. In both cases the intensity shows an initial decrease. In the case shown in Figure 6A this decrease was by a factor of 10 between 0 and $400 \mathrm{~s}$, after which the PL intensity remained low for a considerable time between 400 and 1100 s. Remarkably, after $1100 \mathrm{~s}$ of photoetching, the photoluminescence increased suddenly by over 3 orders of magnitude with respect to the initial intensity and then slowly decayed to an intensity which is still 2 orders of magnitude higher than that of the initial situation. We attribute the initial decrease of the photoluminescence to the removal of the passivating oxide layer, leaving a poorly passivated surface. This experiment was repeated with other wires, and all showed the characteristic initial decrease and subsequent increase in luminescence intensity; an example with a small increase in the PL intensity is shown in Figure 6B. Additionally, we found that the maximum of the luminescence spectrum was blue-shifted by some tens of millielectronvolts simultaneously with the increase of the photoluminescence.

We studied the stability of the luminescence of wires, which showed a considerable increase in their photoluminescence after the photoetching under intense illumination (laser spot $15.9 \mathrm{~kW} /$ $\mathrm{cm}^{2}$ ). We measured the time evolution of the photoluminescence of photopassivated wires after washing and drying under ambient conditions. It was commonly found that the luminescence slowly decreased initially, but became constant at relatively low luminescence intensity. Further research is needed to investigate the origin of this moderate degradation. We observed remarkable photoluminescence intensity fluctuations from single wires on the seconds time scale (Figure 7). We note that these fluctuations were observed only with photoetched wires; a typical time trace of a nonetched wire is also shown in Figure 7 (black trace). The fluctuations in the intensity were correlated to shifts in the peak position. The photoluminescence did not jump between the "off" and "on" states as observed with individual quantum dots, but the amplitude of the jumps varied between 0 and $50 \%$ of the total intensity. This implies that a nonradiative surface center influences a part of the wire (within the exciton diffusion length). Excitons generated in this part recombine nonradiatively, while the excitons generated in another region lead to luminescence. In this view, the fluctuations in the luminescence intensity reflect the spatially random generation/recombination of excitons. Another possibility is that, under intense laser illumination, defects are randomly generated and annealed.

\section{Conclusions}

We have shown that InP nanowires can be passivated by a photoetching process to increase the luminescence efficiency considerably. The enhancement of the photoluminescence varies strongly from wire to wire, but enhancement by 1 order of magnitude can often be achieved. The process can be tuned, such that the surface is passivated with improved photoluminescence, without a noticeable reduction of the wire diameter. Moreover, by the use of polarized light, wires can be photoetched and passivated selectively by proper orientation of the polarization vector. For instance, this could be used to remove wires with an undesirable orientation for device applications. Photoetched wires that are passivated with TOPO ligands do not show a completely stable luminescence under intense excitation in ambient. The time traces show interesting fluctuations in intensity, similar to the on-off fluctuations observed with quantum dots. The correlation between the intensity and the wavelength fluctuations will be studied in more detail in the near future.

Acknowledgment. This work is part of the research program of the "Stichting voor Fundamenteel Onderzoek der Materie (FOM)", which is financially supported by the Nederlandse Organisatie voor Wetenschappelijk Onderzoek (NWO).

JA0518600 